\title{
The effect of a thermal renal denervation cycle on the mechanical properties of the arterial wall
}

\author{
Alan A. Hopkins ${ }^{a, b, 1}$, William S. Sheridan ${ }^{a, b, 1}$, Faisal Sharif ${ }^{a, c}$, Bruce P. Murphy ${ }^{a, b, d, *, 2}$ \\ a Trinity Centre for Bioengineering, Trinity Biomedical Sciences Institute, 152-160 Pearse Street, Trinity College Dublin, Dublin 2, Ireland \\ ${ }^{\mathrm{b}}$ Department of Mechanical and Manufacturing Engineering, School of Engineering, Trinity College Dublin, Dublin 2, Ireland \\ ${ }^{c}$ Regenerative Medicine Institute, National Centre for Biomedical Engineering Science, National University of Ireland, Galway, Ireland \\ ${ }^{\mathrm{d}}$ Advanced Materials and Bioengineering Research (AMBER) Centre, Trinity College Dublin, Dublin, Ireland
}

\section{A R T I C L E I N F O}

\section{Article history:}

Accepted 25 September 2014

\section{Keywords:}

Renal denervation

Arterial wall

Mechanical properties

Hypertension

Renal artery

\begin{abstract}
A B S T R A C T
The aim of this study was to determine the effect that a thermal renal denervation cycle has on the mechanical properties of the arterial wall. Porcine arterial tissue specimens were tested in three groups: native tissue, decellularized tissue, decellularized with collagen digestion (e.g. elastin only). One arterial specimen was used as an unheated control specimen while another paired specimen was subjected to a thermal cycle of $70{ }^{\circ} \mathrm{C}$ for $120 \mathrm{~s}(n=10)$. The specimens were subjected to tensile loading and a shrinkage analysis. We observed two key results: The mechanical properties associated with the elastin extracellular matrix (ECM) were not affected by the thermal cycle. The effect of the thermal cycle on the collagen (ECM) was significant, in both the native and decellularized groups the thermal cycle caused a statistically significant decrease in stiffness, and failure strength, moreover the native tissue demonstrated a $27 \%$ reduction in lumen area post exposure to the thermal cycle. We have demonstrated that a renal denervation thermal cycle can significantly affect the mechanical properties of an arterial wall, and these changes in stiffness and failure strength were associated with alterations to the collagen rather than the elastin extracellular matrix component.
\end{abstract}

(c) 2014 Published by Elsevier Ltd.

\section{Introduction}

Catheter based renal denervation (RDN) has recently been developed, and successfully adopted, as a procedure to control high blood pressure in patients that are resistant to lifestyle changes and antihypertensive medical therapy. The procedure has demonstrated efficacy and good mid-term safety data in a resistant hypertensive patient cohort. Whereby initial clinical trials demonstrate that office based systolic blood pressure can be substantially reduced and adverse procedure-related events minimised (Krum et al., 2009; Investigators, 2011; Esler et al., 2012). The reduction in office based blood pressure has been demonstrated to be durable, with patients sustaining a reduction in blood pressure out to three years (Krum et al., 2013). The devices employed in this procedure use thermal energy as the

\footnotetext{
* Corresponding author at: Trinity Centre for Bioengineering, Trinity Biomedica Sciences Institute, 152-160 Pearse Street, Trinity College Dublin, Dublin 2, Ireland E-mail addresses: ahopkin@tcd.ie (A.A. Hopkins), sheridaw@tcd.ie (W.S. Sheridan), Faisal.Sharif@nuigalway.ie (F. Sharif), bruce.murphy@tcd.ie (B.P. Murphy).

${ }^{1}$ Tel.: + 3531896 3678; fax: +3531679 5554 .

2 Tel.: +35318968503.
}

method to obliterate the sympathetic renal nerves. In some instances a single radio frequency ( $R F$ ) electrode is utilised at multiple sites (Krum et al., 2009), or multiple electrodes are used simultaneously (Worthley et al., 2013), or one larger spiral electrode can be employed (Ormiston et al., 2013), or catheter-based high-frequency ultrasound transducers can be used (Mabin et al., 2012). One feature common to all of the devices/protocols is the application of a thermal cycle to the area surrounding the renal artery, commonly involving temperatures of approximately $70{ }^{\circ} \mathrm{C}$ for a time period of 1-2 min.

As a consequence of the thermal cycle the arterial wall is exposed to an elevated temperature; however, limited pre-clinical documentation of alteration to the arterial wall's structure post thermal ablation is available in the literature. In a study by Rippy et al. (2011) porcine histology results at six months post ablation are depicted, the histology images detail areas of medial fibrosis and medial proteoglycan deposition, demonstrating that some form of thermal injury or remodelling persists within a healthy vessel six months post thermal ablation. Clinical findings demonstrate that significant vasospasm is present post catheter based renal denervation (Templin et al., 2013), in a study by Templin and co-workers Optical Coherence Tomography (OCT) was utilised to identify: vasospasm induced by the EnligHTN ${ }^{\mathrm{TM}}$ catheter (St. Jude Medical), 
and vessel wall oedemas that were caused by the Simplicity catheter (Medtronic). Additionally, there have been cases of stenosis occurring post RDN, in one patient two stenotic sites were observed in a renal artery five months post the denervation procedure (Kaltenbach et al., 2012), separately a second patient presented with a hemodynamically relevant stenosis near the ostium six months post catheter based renal denervation (Vonend et al., 2012). Potentially these findings suggest that the wall of the artery may be detrimentally modified post thermal catheter based renal denervation.

The effect of elevated temperatures on the arterial wall's extracellular matrix may play a role in altering the functionality of a healthy vessel. The medial layer of the arterial wall is exposed to elevated temperatures, this in turn can affect the major structural proteins (elastin and collagen) or/and the smooth muscle cells. Previously it has been shown that a significant level of medial necrosis has been observed when radio-frequency energy was applied to heat a porcine arterial wall to $70^{\circ} \mathrm{C}$ for $60 \mathrm{~s}$ (Fram et al., 1993). The depth of heat necrosis in this study was measured as $1031 \mu \mathrm{m}$; demonstrating that substantial medial layer smooth muscle cell death may occur during renal denervation procedures that use temperatures in the range of $70^{\circ} \mathrm{C}$. Additionally, there is a fine balance associated with the smooth muscle cells and the extracellular matrix components of the vessel wall, whereby small collagen fibres and large collagen bundles as well as fenestrated elastin sheets all interact with the smooth muscle cells to form a functioning arterial wall (O'Connell et al., 2008). Any disruption to this extracellular matrix architecture may cause vessel wall dysfunction. The denaturation temperature of collagen has been reported in a number of studies as being in the range of $50-60{ }^{\circ} \mathrm{C}$ (Gross, 1964; Venkatasubramanian et al., 2010). These results would imply that some form of reduction in mechanical stability of the arterial wall would occur post a renal denervation thermal cycle, as it contains significant amounts of collagen-the main structural element. Elastin denaturation on the other hand does not seem to be affected by the temperature range associated with thermal renal denervation procedures (Lillie et al., 1994). In these prior studies the overall effect on the arterial wall structural integrity has not been documented, and only isolated aspects of the mechanics or denaturation of the ECM components have been investigated with respect to increases in temperature.

We hypothesised that heating an arterial wall to a temperature level associated with thermal renal denervation procedures will affect the structural integrity of the arterial wall. To test this hypothesis we designed and conducted a series of experiments that heated and subsequently mechanically tested the arterial tissue post heating to determine the effect on the mechanical properties.

\section{Methods}

\subsection{Tissue harvest}

Porcine carotid arteries were freshly harvested from 70 to $90 \mathrm{~kg}$ pigs in a local abattoir (Lislin Meats Ltd, Mullagh, Co. Cavan, Ireland). Common carotid arteries in the 5-7 mm outer diameter range were excised. All arteries were returned to the laboratory and stored in phosphate buffered saline (PBS) on ice. Samples were additionally prepared by removing excess connective and adventitial tissue and cut into approximately $30 \mathrm{~mm}$ long segments. All specimens were then frozen in PBS at $-20{ }^{\circ} \mathrm{C}$ for later use. Specimens were divided into three groups: native, decellularized, and decellularized with collagen digestion. This allowed us to examine the effects of the thermal cycle on the native tissue, the isolated extracellular matrix, and a scaffold that closely resembles an elastin-only scaffold.

\subsection{Decellularization}

To investigate the effect of the temperature rise on the isolated extracellular matrix components, we removed the cells from the tissue with a decellularization protocol. Decellularization was carried out as previously described (Sheridan et al., 2012). In brief, the protocol consists of an enzymatic digestion and detergent extraction. Constructs were immersed in de-ionized water for $24 \mathrm{~h}$ at $4{ }^{\circ} \mathrm{C}$ under rotational agitation and subsequently incubated in 0.05\% Trypsin with $0.02 \%$ EDTA (Sigma-Aldrich, Ireland) for $1 \mathrm{~h}$ at $37{ }^{\circ} \mathrm{C}$. After a short rinse in PBS to remove excess trypsin, the samples were placed in a solution of $2 \%$ Triton X-100 and $0.8 \%$ ammonium hydroxide (Sigma-Aldrich, Ireland) in de-ionized water for $72 \mathrm{~h}$ at $4{ }^{\circ} \mathrm{C}$ under rotational agitation. The solution was changed daily. A final wash sequence of $48 \mathrm{~h}$ in de-ionized water was undertaken to remove any residual chemicals from the constructs.

\subsection{Collagen digested tissue}

To investigate the effect of the temperature rise on arterial elastin we removed the collagen from the tissue in a secondary process that occurred after decellularization. In this process sodium hydroxide $(\mathrm{NaOH})$ was used to digest the collagen. The additional step involved subjecting the samples to sonication in $1.5 \mathrm{M} \mathrm{NaOH}$ for $180 \mathrm{~min}$ prior to a final wash sequence of $48 \mathrm{~h}$ in de-ionized water under constant agitation, to remove excess chemicals; the de-ionized water was changed after $24 \mathrm{~h}$.

\subsection{Specimen preparation and thermal cycle}

Specimens were prepared by firstly thawing the retrieved arteries and subsequently isolating a 4-6 mm diameter, and $30 \mathrm{~mm}$ length segment of an artery. This segment was then cut into two equal $4 \mathrm{~mm}$ length specimens, these specimens were cut from adjacent sections in the centre of the segment length and used as paired controls in the mechanical testing phase. A specially designed tool was used to ensure every specimen was always cut to $4 \mathrm{~mm}$ in length. Subsequently one of the paired specimens was subjected to a thermal cycle. The thermal cycle consisted of immersion in a temperature controlled water bath at $70{ }^{\circ} \mathrm{C}$ for $120 \mathrm{~s}$. This thermal cycle mimics a thermal renal denervation cycle.

\subsection{Mechanical testing}

Uniaxial tensile tests were carried out to determine the tensile mechanical response of the native, decellularized and collagen digested tissues using a Zwick tensile testing machine (Zwick Z005, Roell, Germany). The following groups ( $n=10$ each) were tested: (i) native arterial tissue, (ii) decellularized tissue, (iii) collagen digested tissue. Each of the three groups consisted of 10 unheated specimens and 10 specimens exposed to the thermal cycle. Each specimen was tensile tested to failure and the force was recorded using a $100 \mathrm{~N}$ load cell. Custom made grips, a PBS bath and video extensometry was used to determine the specimen's displacement, see Sheridan et al. for a more detailed description of the tensile test experimental set-up (Sheridan et al., 2012). True stress $(\sigma)$ and true strain $(\varepsilon)$ were utilised as the investigated parameters.

\subsection{Shrinkage}

Specimen shrinkage was recorded by capturing an image of the specimen's inner lumen dimensions prior to mechanical testing. All samples were mounted on the grips and subjected to a small force of $0.5 \mathrm{~N}$ and an image was subsequently acquired of the cross section of the specimen. The inner circumference was determined from the image, and consequently the area was calculated, this was conducted for all the unheated and heated paired specimens.

\subsection{Histology and scanning electron microscopy (SEM)}

The specimens that were investigated by histological analysis were embedded in paraffin wax in an automatic tissue processor (ASP300, Leica, Germany). All samples were sectioned longitudinally using a rotary microtome (Leica microtome, Leica, Germany). $7 \mu \mathrm{m}$ sections were cut and collected on glass slides and subsequently washed through a graded series of ethanol from 100 to $70 \%(\mathrm{v} / \mathrm{v}$ ) Samples of native, decellularized tissue, and decellularized with collagen digestion were stained using Picrosirius Red. To further verify cell removal, sections were stained with diamidino-2-phenylindole (DAPI). Sections were dehydrated and cleaned in ascending concentrations of ethanol and xylene before coverslips were mounted (DPX mountant, BDH). Specimens viewed in the SEM were fixed in 2.5\% buffered glutaraldehyde for $24 \mathrm{~h}$. These specimens maintained their cylindrical configuration. Each specimen was then subjected to sequential washes of $30 \%, 50 \%$, $70 \%, 90 \%$ and $100 \%$ ethanol for 15 min with the final wash performed three times The dried samples were mounted on an aluminium stub and sputter coated with gold. An SEM (Zeiss Ultra Plus, Germany) was used to view the ultrastructure of the medial layer of the control specimens and specimens subjected to a thermal cycle.

\subsection{Statistical analysis}

All comparisons were conducted utilising paired tests on the normally distributed date, whereby each control unheated specimen was associated with a 
heated specimen. A students $T$-test was used to test significance between each group separately and results with $p<0.05$ were considered statistically significant Box plots were used to present the distributions of the data. Each box displays interquartile range (IQR) with median and outliers (defined as median $\pm 1.5 * \mathrm{IQR}$ ) are shown with individual dots.

\section{Results}

\subsection{Mechanical testing}

The mechanical tests produced stress versus strain graphs, and additional information that relates to the tissue's mechanical integrity. The average stress-strain graphs for the native tissue group revealed that the tissue exposed to the thermal cycle exhibited an average behaviour that was shifted to the left of the control unheated group, see Fig. 1A. This shift indicates that some form of alteration to the collagen component has taken place as a result of exposure to the heating cycle. Earlier engagement of the collagen stiff region can be observed in the heated specimen's graph, this is detected by the "steepness" of the heated curve occurring at a lower strain.

The results of the experiments that examined the effect of heating on the arterial wall's extracellular matrix components (e.g. the decellularized tissue) revealed that the mechanical response of the heated decellularized tissue is significantly altered in comparison to the control unheated decellularized tissue, this can be observed as a considerably weaker response in the stress strain data, see Fig. 1B. This would indicate that the structural integrity of the collagen element has been destroyed; this result can be further backed-up by the collagen digested tissue data, whereby the response of the decellularized heated specimen's curve is identical to the collagen digested curves shown in Fig. 1C, demonstrating that no collagen mechanical response is present in the heated decellularized specimens. The elastin scaffolds (e.g. collagen digested tissue) demonstrated that no noticeable difference was observed in the heated versus the unheated groups, see Fig. 1C, indicating that any change in the mechanical properties of the arterial wall post a thermal cycle is related to alterations within the collagen element of the arterial wall's extracellular matrix.

The effect of the thermal cycle on the stiffness of the collagen controlled area of the stress-strain curve was determined by plotting the modulus of the collagen dominant phase for the paired specimens for the three groups. For details of the method employed to determine the collagen dominant stiffness phase of the stress strain curve see Sheridan et al. (2012). The native tissue demonstrated a pattern of reduced collagen stiffness between the paired specimens; while the decellularized tissue demonstrated a very sharp reduction in the stiffness, and the collagen digested tissue demonstrated no change, see Fig. 1D-F. Noticeably the reduction in stiffness was generally matched with the paired specimens and was not just an artefact of significant outliers (see Fig. 1D-F). The box plots shown in Fig. 1G-I reveal the distribution of the stiffness values for all three groups, it can be noted that there is a statistically significant difference between the unheated native and the heated native tissue and the unheated decellularized and the heated decellularized tissue sub-groups.

The failure strength of the native tissue was reduced when exposed to the thermal cycle, moreover the failure strength of the decellularized tissue that was exposed to the thermal cycle was substantially reduced, while the elastin only tissue demonstrated no difference in failure strength, see Fig. 1(J-L).

\subsection{Shrinkage analysis}

The specimens were assessed to determine the level of tissue shrinkage after exposure to the thermal cycle. The native tissue displayed the largest degree of shrinkage when exposed to the thermal cycle, with a statistically significant difference associated with the heated group versus the control group $(p<0.05)$. The thermal cycle caused a $27 \%$ decrease in lumen area versus the unheated group in the native tissue group. While in the case of the decellularized tissue and the decellularized with collagen digestion tissue there was no significant shrinkage observed, see Fig. 2.

\subsection{Histology and SEM}

Histological and SEM analysis of the native unheated tissue revealed a presence of thick collagen fibres and an un-crimped medial layer, while the heated specimens reveal that the thick collagen fibres are absent in the SEM images and the collagen fibres appear heavily crimped when viewed under in the histological images, see Fig. 3. In the case of the decellularized and decellularized with collagen digestion collagen fibres are difficult to observe in both the heated and unheated groups, and when observed are thin in their nature, resembling structures that would support minimal mechanical loads (data not shown).

\section{Discussion}

A number of questions have arisen with respect to utilising a thermal approach to ablate the sympathetic nervous system that surrounds the renal arteries. Namely, what is the long term effect on the arterial wall: is it detrimental or does it have limited consequences on the renal artery? In this study we have been able to demonstrate two phenomena: the structural properties associated with the elastin component of the extracellular matrix were not affected by the thermal cycle. This was observed in the decellularized collagen digested group (i.e. elastin only), whereby no difference is observed in the average stress-strain response, modulus, or failure strength after the application of a thermal cycle. Moreover the shrinkage response is identical in this group when the control experiment is compared to the specimens exposed to the thermal cycle. This minimal response may have implications in the formation of aneurysms, as it has been shown in model systems that degradation of the elastin extracellular matrix component can lead to the development of aneurysms (Anidjar et al., 1990; Bi et al., 2013). To this end, the effect of the thermal cycle on the elastin mechanical properties is minimal, and potentially the formation of renal artery aneurysms may not be a concern with long term clinical outcomes of thermal renal denervation procedures.

The effect of the thermal cycle on the collagen component of the extracellular matrix is significant. In the isolated extracellular matrix specimens a statistically significant difference in the stiffness (i.e. the modulus) of the tissue is present, whereby the average collagen stiffness is only $16 \%$ of the control specimens highlighting that the thermal cycle significantly degrades the collagen structure in these specimens. This potentially is an anticipated result, as the temperature at which collagen degrades is reported as being in the range of $50-60{ }^{\circ} \mathrm{C}$ (Gross, 1964; Venkatasubramanian et al., 2010). The degradation in the collagen stiffness is not as pronounced in the native specimens, whereby the collagen retains $60 \%$ of it mechanical stiffness in comparison to the unheated controls. Potentially this stiffness reduction may have negative implications if repopulation of smooth muscle cells occurs, as Brown et al. (2010) demonstrated that a reduction to $61 \%$ of the native tissue's stiffness value caused vascular smooth muscle cells not to survive overnight in tissue culture on these reduced stiffness substrates. However, they also demonstrated that proliferation on an even stiffer substrate was far greater, a 4.5 fold increase in proliferation was observed in 
A

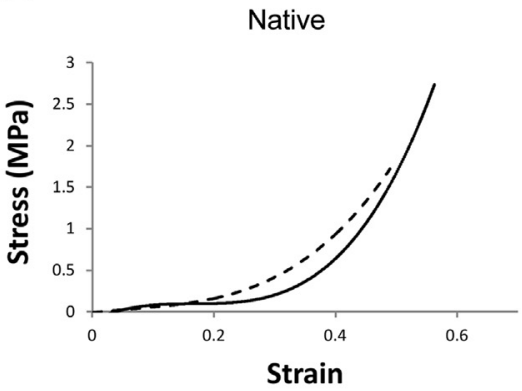

D

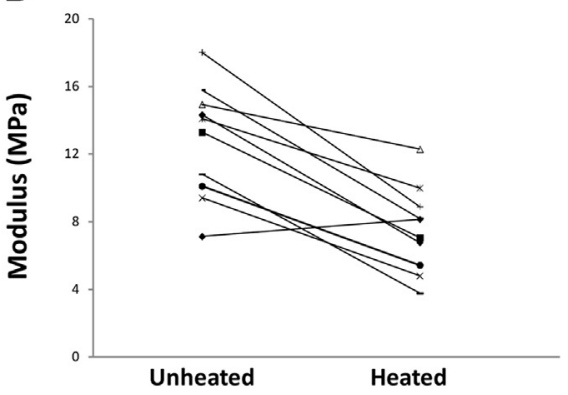

G

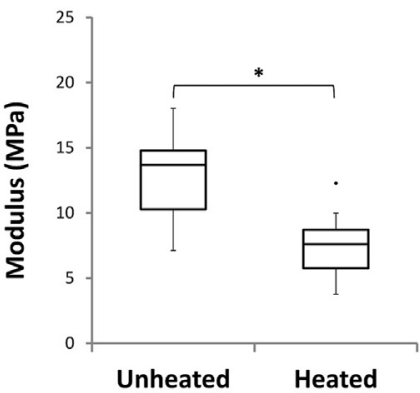

$J$

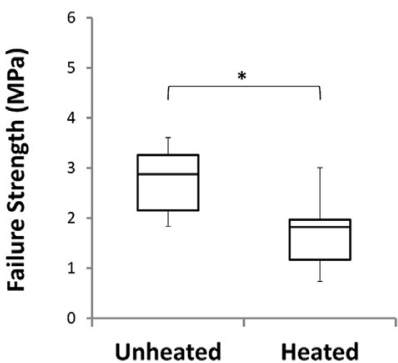

B

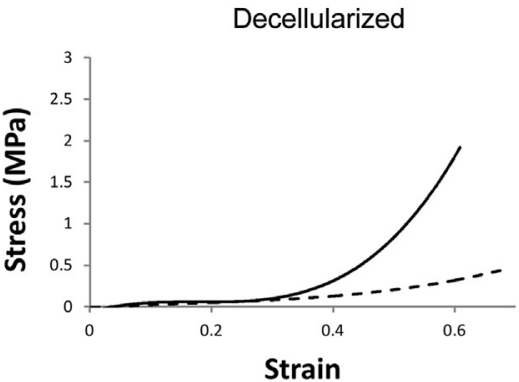

$E$

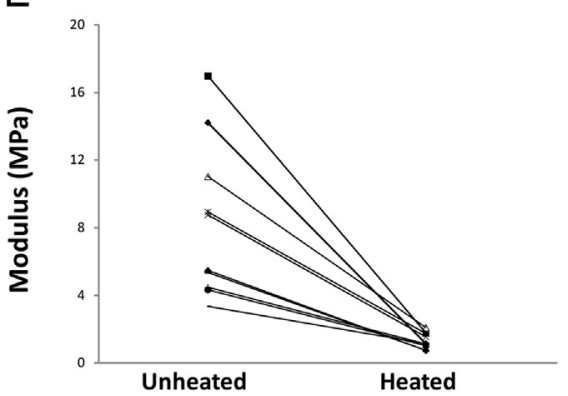

$\mathrm{H}$

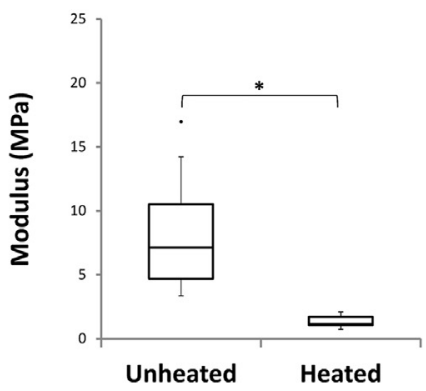

K

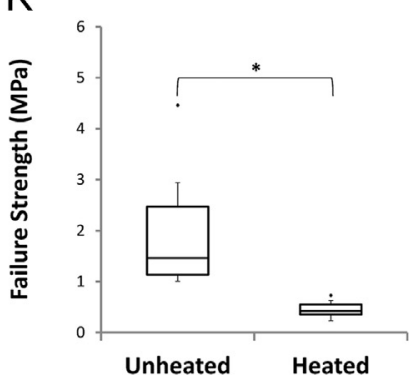

C
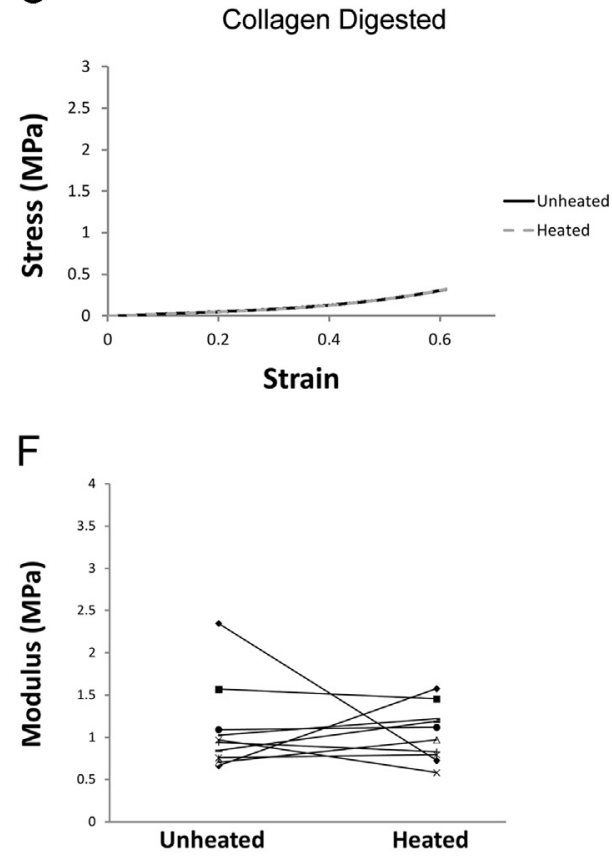

I

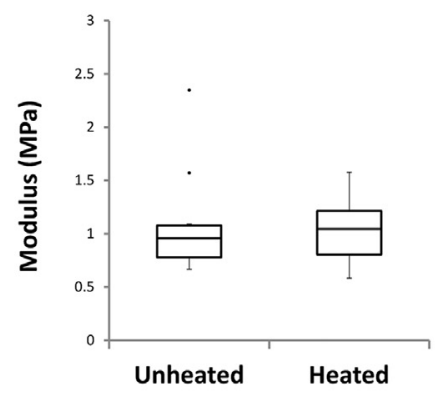

$\mathrm{L}$

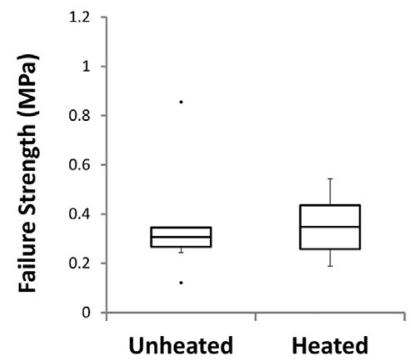

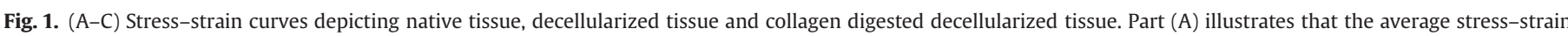

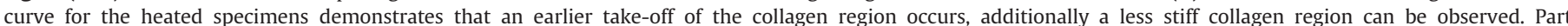

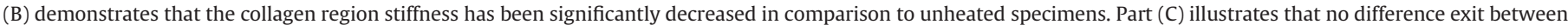

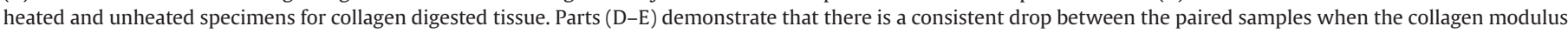

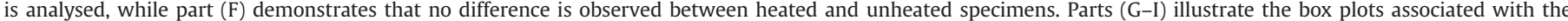

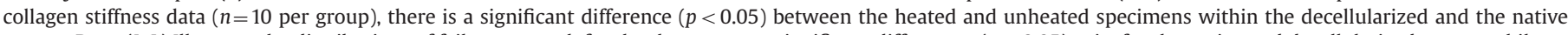

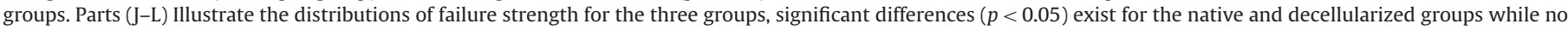
difference exists in the collagen digested group.

comparison to the native stiffness mimicking substrate. Potentially the Brown et al. result implies that SMC's "like" a certain substrate stiffness and if this is altered their phenotype can be substantially changed.

The application of the thermal cycle reduced the average failure strength of the native tissue and the decellularized tissue by $38 \%$ and $77 \%$ respectively. The physiological applicability of these results should not be overstated, as arterial structures generally operate well below their failure stresses. However, in a small number of cases some form of vascular reconstruction may take place and there may be inherently weaker structures present to hold sutures, thus in this case failure strength may be important in patients that require some form of reconstruction such as surgical aneurysm repair.

One other important aspect that may affect immediate results is the shrinkage associated with the tissue. We noted 


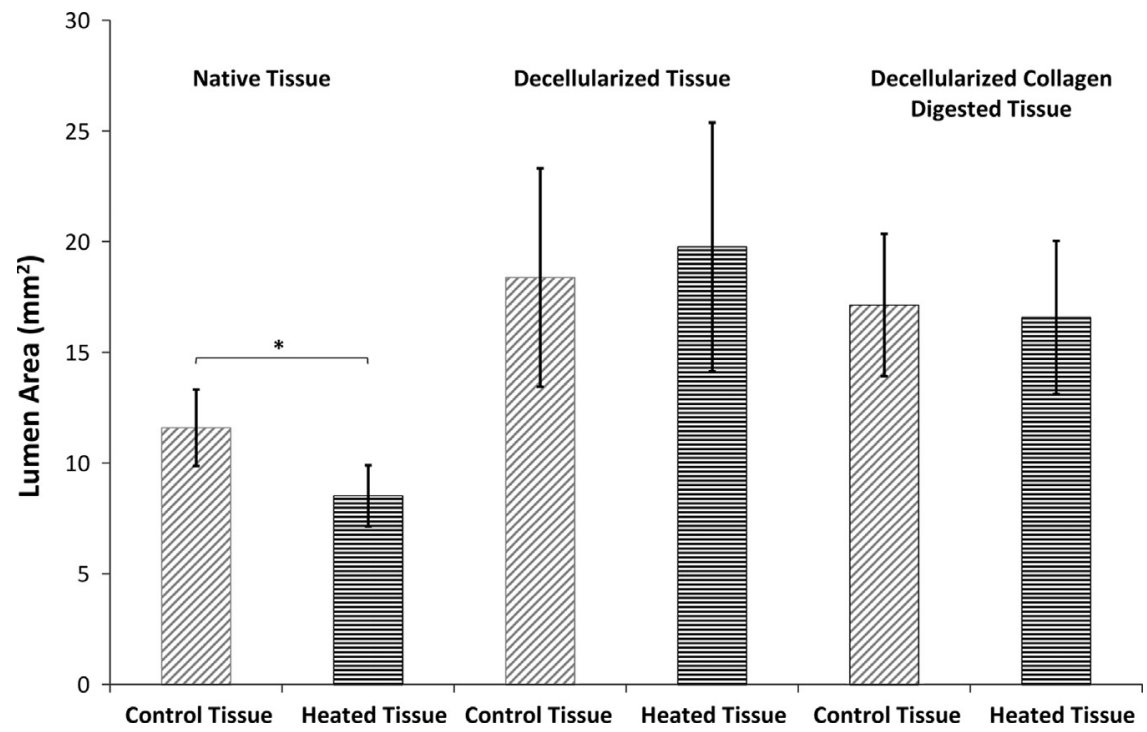

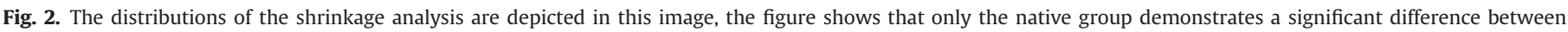
heated and unheated specimens $(p<0.05)$.
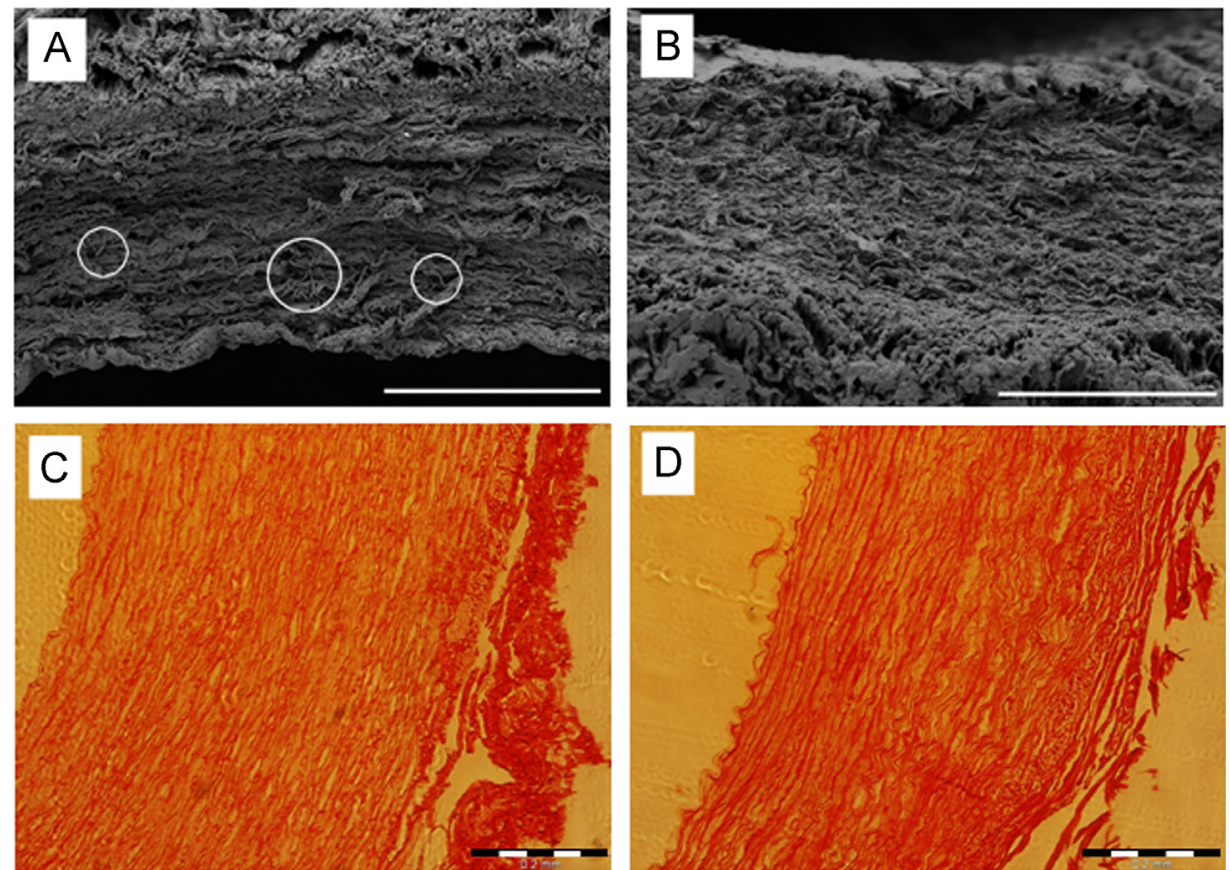

Fig. 3. (A) Depicts a scanning electron microscope image of an unheated native specimen, the white circles highlight intact large collagen bundles, in the heated native image (B) these large collagen bundles are not present. Image panel (C) shows an unheated native H\&E stained image of an artery, while part (D) depicts a heated specimen, differences between the two images include changes in the collagen fibres-in the heated specimens the collagen appears heavily crimped. All scale bars equate to $200 \mu \mathrm{m}$.

no statistical difference between either the elastin component or the extra cellular matrix groups between heated and unheated specimens. However when native tissue was subjected to a thermal cycle a $27 \%$ decrease in lumen area was observed. This shrinkage may have a limited effect on flow for a number of the devices that utilise smaller probes, as areas of shrinkage will be confined to small regions. However for devices that use larger probes or devices that induce circumferential heated zones the issue of shrinkage has an implication on flow through the vessel, and could lead to a reduced volume of blood flowing to the kidneys until the shrinkage can be remodelled. It has been shown previously that applying a thermal cycle to a tissue containing a collagen structure can cause homogenous shrinkage, whereby Rahman et al. (2010) report that the mitral annulus in an ovine model can be reduced by $21.7 \%$ by the application of a thermal cycle of $65{ }^{\circ} \mathrm{C}$ for $50-60 \mathrm{~s}$. This result is in agreement with our results presented in this study. The mechanism of shrinkage may be similar to the hypothesis presented by Rahman et al. whereby only partial denaturation and remodelling occurs, however in the ECM only scaffold a larger denaturation effect may occur and the shrinkage effect is eliminated.

There are a number of limitations to this study, namely we used porcine arterial tissue and a water bath to heat the tissue. To mitigate any risks of achieving non-clinically relevant results we choose porcine tissue as it has been previously stated that porcine 
models of stenosis closely resemble human disease states (Lerman et al., 1999), and additionally porcine models are used as the gold standard model for in vivo stent evaluation(Swindle et al., 2012). Additionally, it has been reported in vascular and cardiovascular tissues that collagen density and orientation can change with age, and this can result in subsequent mechanical property alterations (Aronow, 1999; Martin et al., 2011).The comparison of young healthy porcine tissue to aged human tissue will limit exact comparison of the results, as reported in this study. However, as our study is a comparative study, we would assume the application of a thermal cycle to aged human tissue may result in a similar relative drop in stiffness in comparison to control aged unheated human tissue. Moreover, we utilised a water bath environment to deliver the thermal energy rather than an RF or ultrasound method, and we assumed that the rate of temperature rise was equivalent across the various modes of heating. There may be some small errors here however we expect that they would not affect the results as we demonstrate similar results in the shrinkage study to Rahmanet al. (2010), and their protocol is approximately half the time period of ours, demonstrating that small changes in the rate of heating may not have large effects on the results. We did not test in the longitudinal direction and limited our experimental program to the circumferential direction only, potentially the circumferential test results could be used to scale the longitudinal stiffness for tissues that are exposed to a thermal cycle, this may be important for researchers utilising computational models of arterial tissue that is exposed to a thermal cycle.

In conclusion we have demonstrated that a renal denervation thermal cycle can significantly affect the mechanical properties of the arterial wall. More, specifically we have shown that a loss in stiffness and failure strength occurs, and our results suggest that this loss in mechanical integrity is due to degradation of the collagen structure. Moreover we observed no change to the mechanical response of the elastin extracellular matrix component. One additional main finding was that we observed an average shrinkage of $27 \%$ occurring in native tissue when exposed to the thermal cycle which can translate into a significant reduction in lumen cross section. The results of this study do not suggest that there are any concerns over safety of thermal renal denervation procedures; however they do highlight that alterations to the extracellular matrix occur within the tissue when a thermal cycle is delivered to an arterial wall and potentially highlight that correctly designed cooling systems would be useful features that would insure that thermal induced damage is reduced.

\section{Conflict of interest statement}

The authors have no conflict of interest.

\section{References}

Anidjar, S., Salzmann, J.L., et al., 1990. Elastase-induced experimental aneurysms in rats. Circulation 82 (3), 973-981.
Aronow, W.S., 1999. The older man's heart and heart disease. Med. Clin. North Am 83 (5), 1291-1303.

Bi, Y., Zhong, H., et al., 2013. Development of a novel rabbit model of abdominal aortic aneurysm via a combination of periaortic calcium chloride and elastase incubation. PLoS One 8 (7), e68476.

Brown, X.Q., Bartolak-Suki, E., et al., 2010. Effect of substrate stiffness and PDGF on the behavior of vascular smooth muscle cells: implications for atherosclerosis. J. Cell Physiol. 225 (1), 115-122.

Esler, M.D., Krum, H., et al., 2012. Renal sympathetic denervation for treatment of drug-resistant hypertension: one-year results from the Symplicity HTN-2 randomized, controlled trial. Circulation 126 (25), 2976-2982.

Fram, D.B., Aretz, T.A., et al., 1993. In vivo radiofrequency thermal balloon angioplasty of porcine coronary arteries: histologic effects and safety. Am. Heart J. 126 (4), 969-978.

Gross, J., 1964. Thermal denaturation of collagen in the dispersed and solid state. Science 143 (3609), 960-961.

Investigators, S.H., 2011. Catheter-based renal sympathetic denervation for resistant hypertension: durability of blood pressure reduction out to 24 months. Hypertension 57 (5), 911-917.

Kaltenbach, B., Id, D., et al., 2012. Renal artery stenosis after renal sympathetic denervation. J. Am. Coll. Cardiol. 60 (25), 2694-2695.

Krum, H., Schlaich, M., et al., 2009. Catheter-based renal sympathetic denervation for resistant hypertension: a multicentre safety and proof-of-principle cohort study. Lancet 373 (9671), 1275-1281.

Krum, H., Schlaich, M.P., et al., 2013. Percutaneous renal denervation in patients with treatment-resistant hypertension: final 3-year report of the Symplicity HTN-1 study. Lancet.

Lerman, L.O., Schwartz, R.S., et al., 1999. Noninvasive evaluation of a novel swine model of renal artery stenosis. J. Am. Soc. Nephrol. 10 (7), 1455-1465.

Lillie, M.A., Chalmers, G.W., et al., 1994. The effects of heating on the mechanical properties of arterial elastin. Connect. Tissue Res. 31 (1), 23-35.

Mabin, T., Sapoval, M., et al., 2012. First experience with endovascular ultrasound renal denervation for the treatment of resistant hypertension. EuroIntervention 8 (1), 57-61.

Martin, C., Pham, T., et al., 2011. Significant differences in the material properties between aged human and porcine aortic tissues. Eur. J. Cardiothorac. Surg. 40 (1), 28-34.

O'Connell, M.K., Murthy, S., et al., 2008. The three-dimensional micro- and nanostructure of the aortic medial lamellar unit measured using 3D confocal and electron microscopy imaging. Matrix Biol. 27 (3), 171-181.

Ormiston, J.A., Watson, T., et al., 2013. First-in-human use of the OneShot renal denervation system from Covidien. EuroIntervention 8 (9), 1090-1094.

Rahman, S., Eid, N., et al., 2010. Remodeling of the mitral valve using radiofrequency energy: review of a new treatment modality for mitral regurgitation. Cardiovasc. Revasc. Med. 11 (4), 249-259.

Rippy, M.K., Zarins, D., et al., 2011. Catheter-based renal sympathetic denervation: chronic preclinical evidence for renal artery safety. Clin. Res. Cardiol. 100 (12) 1095-1101.

Sheridan, W.S., Duffy, G.P., et al., 2012. Mechanical characterization of a customized decellularized scaffold for vascular tissue engineering. J. Mech. Behav. Biomed. Mater. 8, 58-70.

Swindle, M.M., Makin, A., et al., 2012. Swine as models in biomedical research and toxicology testing. Vet. Pathol. 49 (2), 344-356.

Templin, C., Jaguszewski, M., et al., 2013. Vascular lesions induced by renal nerve ablation as assessed by optical coherence tomography: pre- and postprocedural comparison with the Simplicity(R) catheter system and the EnligHTN multi-electrode renal denervation catheter. Eur. Heart J. 34 (28), 2141-2148.

Venkatasubramanian, R.T., Wolkers, W.F., et al., 2010. Freeze-thaw induced biomechanical changes in arteries: role of collagen matrix and smooth muscle cells. Ann. Biomed. Eng. 38 (3), 694-706.

Vonend, O., Antoch, G., et al., 2012. Secondary rise in blood pressure after renal denervation. Lancet 380 (9843), 778.

Worthley, S.G., Tsioufis, C.P., et al., 2013. Safety and efficacy of a multi-electrode renal sympathetic denervation system in resistant hypertension: the EnligHTN I trial. Eur. Heart J. 34 (28), 2132-2140. 